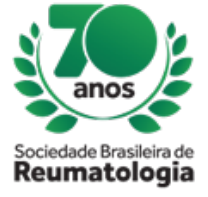

\title{
LUPUS RETINOPATHY AND REFRACTORY SYMPTOMS TO THE TREATMENT OF SYSTEMIC LUPUS ERYTHEMATOSUS OF DIFFICULT CONTROL: A CASE REPORT
}

Ana Olívia Dantas (Universidade Federal do Rio Grande do Norte, Caicó, RN, Brasil), Leoberto Batista Pereira Sobrinho (Universidade Federal do Rio Grande do Norte, Caicó, RN, Brasil), Laura Alícia Morais Lima Oliveira (Universidade Federal do Rio Grande do Norte, Caicó, RN, Brasil), Lauanda Ênia Medeiros Rocha (Universidade Federal do Rio Grande do Norte, Caicó, RN, Brasil), Lara Valeska Medeiros Rocha

(Universidade Federal do Rio Grande do Norte, Caicó, RN, Brasil), Humberto Cabral Oliveira Filho

(Universidade Federal do Rio Grande do Norte, Caicó, RN, Brasil), Breno Vinícius Dias Souza

(Universidade Federal do Rio Grande do Norte, Caicó, RN, Brasil), Heron Alves Vale (Universidade Federal do Rio Grande do Norte, Caicó, RN, Brasil), Emilie Queiroga Queiroga (Universidade Federal do Rio Grande do Norte, Caicó, RN, Brasil), Pedro Augusto Dias Timoteo (Universidade Federal do Rio Grande do Norte, Caicó, RN, Brasil)

\section{BACKGROUND}

Systemic lupus erythematosus (SLE) is a chronic inflammatory disease that affects multiple organs and systems. Evolving with periods of activity and remission, and the treatment used although effective can cause several secondary effects.

\section{CASE REPORT}

A 38-year-old female patient diagnosed with SLE, complains of edema in the metacarpophalangeal joints, bilateral arthralgia of an inflammatory condition, and morning stiffness. History of edema in lower limbs, dysuria, oral ulcers, and malar erythema, using hydroxychloroquine $400 \mathrm{mg}$, prednisone $5 \mathrm{mg}$, and methotrexate $10 \mathrm{mg}$. The management was the addition of folic acid $5 \mathrm{mg}$, duoflan IM in a single dose and complete biochemistry. At follow-up, the patient reported decreased visual acuity and presented laboratory tests with FAN 1-160, dense fine nuclear speckles, anti-DNA reagent, anti-LA reagent, PCR 24, TGO 144 and TGP 122. Methotrexate was suspended because of a possible association with liver injury, and the patient was sent to an ophthalmologist. A posteriori, the patient complained of polyarthralgia 15 days ago, associated with a low fever, with new exams showing ophthalmologic report of lupus retinopathy with hypermetropy; VDRL 1-128, positive IGG and negative IGM for cytomegalovirus, toxoplasmosis, and herpes, the patient was sent to infectologists. After 22 days of hospitalization, the patient returns with a diagnosis of acute lupus, secondary to syphilis infection, and also fibromyalgia; the management was to increase the dose of prednisone to $40 \mathrm{mg}$ with subsequent reduction to $5 \mathrm{mg}$, maintaining the hydroxychloroquin, and fluoxetine for fibromyalgia. After one year of treatment, the patient returns to the office complaining of chronic diarrhea for 8 months, with foul, massive and yellowish feces associated with fecal incontinence, evacuating 4 to 5 times a day, associated with intense abdominal pain in the left flank with irradiation to the right, in colic, that improves at the end of the defecation. She also presented a weight gain of $20 \mathrm{~kg}$ in the last 5 months, adynamia and myalgia. Colonoscopy was requested to rule out other etiologies but kept the hydroxychloroquin $400 \mathrm{mg}$ and the reduction of prednisone.

\section{CONCLUSION}

The present case showed an atypical presentation of lupus vasculitis, attacking retinal vessels, and intensified by hydroxychloroquin. In addition, the antimalarials, corticosteroids, and immunosuppressants promoted the majority of symptoms such as diarrhea, headache, vomiting, dizziness, muscle weakness, besides the risk of infections, showing the variability of SLE manifestations, and the symptoms refractory of drugs. 